\title{
Entendendo o pacto pela saúde na gestão do SUS e refletindo sua implementação ${ }^{1}$
}

\section{Understanding the health care pact in the management of SUS, and reflecting upon its importance}

\section{Interpretando el pacto por la salud en la gestión del SUS y el impacto de su implementación}

\author{
Rosani Ramos Machado', Eliani Costa", Alacoque Lorenzini Erdmann"', \\ Gelson Luiz de Albuquerque ${ }^{\mathrm{I}}$, Ângela Maria Blatt Ortiga ${ }^{\vee}$
}

\begin{abstract}
${ }^{1}$ Artigo elaborado na Disciplina Administração em Enfermagem e Saúde do Programa de PG ENF UFSC nível de Doutorado em Enfermagem, ministrada pelos professores Gelson Luiz de Albuquerque e Alacoque Lorenzini Erdmann.

' Enfermeira da Secretaria do Estado da Saúde/SC. Docente da Universidade do Vale do Itajaí (SC). Doutoranda do Programa de Pós Graduação de Enfermagem da UFSC. Email: rosaniramos@terra.com.br.

"Enfermeira da Secretaria de Estado da Saúde / Instituto de Psiquiatria (SC). Membro do Grupo de Estudos de História do Conhecimento da Enfermagem (GEHCE). Doutoranda do Programa de Pós Graduação de Enfermagem da UFSC. Email: elianicosta10@terra.com.br.

II' Enfermeira. Doutora em Filosofia da Enfermagem. Professora Titular da UFSC. Pesquisadora e Representante da Área da Enfermagem no CNPq. Coordenadora do Grupo de Estudos e Pesquisas em Administração de Enfermagem e Saúde. Email: alacoque@newsite.com.br.

IV Enfermeiro, Doutor em Filosofia da Enfermagem, Professor Adjunto da UFSC, Pesquisador do GEPADES - Grupo de Estudos e Pesquisas em Administração de Enfermagem e Saúde. Email: gelsonalbuquerque@yahoo.com.br.

${ }^{\vee}$ Enfermeira da Secretaria do Estado da Saúde/SC. Docente da Universidade do Vale do Itajaí (SC). Mestre em Saúde Pública pela UFSC. Email: angelablatt@univali.br.
\end{abstract}

\section{RESUMO}

Este texto objetiva abordar a concepção do Pacto pela Saúde, ainda que de forma breve, de modo a repassar esta informação aos profissionais da saúde, acompanhada de reflexões e questionamentos. O Pacto pela Saúde é um instrumento que vai produzir mudanças significativas nas normativas do Sistema Único de Saúde (SUS) e comporta três dimensões: Pacto pela Vida, Pacto em Defesa do SUS e Pacto de Gestão. Visa qualificar a gestão pública do SUS para maior efetividade, eficiência e qualidade, e implicam em mudança na descentralização, regionalização, mecanismos de gestão regional e no financiamento do SUS. Levanta-se a posição dos colegiados de secretários municipais e estaduais sobre a implantação do pacto. Em seu processo apresentam-se alguns questionamentos ou dúvidas e enfatizam-se a importância de fortalecer a gestão do SUS, conceitos presentes na Constituição Federal e na Lei Orgânica da Saúde.

Descritores: Sistema Único de Saúde; Planejamento em saúde; Descentralização.

\section{ABSTRACT}

This text attempts to briefly outline the conception of the Health Care Pact, in such a way that it passes along this information to health care professionals, together with reflections and questions. The Health Care Pact is an instrument that will produce significant change in the norms of the National Health Care System (Sistema Único de Saúde - SUS) and is composed of three dimensions: Pact with Life, Pact in Defense of SUS, and Management Pact. Gets up the position of the municipal and state secretaries' colegiados on the implantation of the pact. Elt looks to qualify public administration of the SUS for greater effectiveness, efficiency, and quality. It implies a change towards decentralization, regionalization, mechanisms for regional administration, and in financing the SUS. This study engages in dialogue with two managers involved with the process and references the need for implementing these pacts, although they are yet little-known and complex. In this process, this article presents some questions or doubts, emphasizing the importance for strengthening the management of the SUS, concepts which are present in the Brazilian Constitution and in the Brazilian Structural Law of Health Care (Lei Orgânica da Saúde).

Descriptors: National Health System (BR); Descentralization; Health planning.

\section{RESUMEN}

Este texto propone el abordaje de la concepción del Pacto por la Salud, aunque en forma escueta, para poder trasmitirle esta información a los profesionales del ámbito de la salud, incluyendo reflexiones y cuestionamientos. El Pacto por la Salud es un instrumento que producirá cambios significativos en las normativas del Sistema Único de Salud (SUS) y contempla tres aspectos: Pacto por la Vida, Pacto en Defensa del Sus y Pacto de Gestión. Apunta a la calificación de la gestión pública del SUS para una mayor efectividad, eficiencia y calidad, e implican cambios en la descentralización, regionalización, mecanismos de gestión regional y en el financiamiento del SUS. Cabe destacar la Posición de los colegiados de secretarios municipales y provinciales sobre la implantación del pacto. En su proceso se presentan algunos cuestionamientos o dudas y se enfatiza la importancia de fortalecer la gestión del SUS, conceptos estos presentes en la Constitución Federal y en la Ley Orgánica de la Salud.

Descriptors: Sistema Único de Salud; Descentralización; Planificación en salud. 


\section{CONSI DERAÇÕES I NICIAIS SOBRE O PACTO PELA SAÚdE NO BRASI L}

Este texto objetiva abordar inicialmente as dimensões do Pacto pela Saúde e em seguida apontar como ocorreu a adesão pelos seus interloutores.Trata-se de um conteúdo que ainda se apresenta em forma de normativas e portarias expedidas pelo Ministério da Saúde ou pelas CIB Comissão Intergestores Bipartite ou CIT - Comissão Intergestores Tripartite. Estas informações apesar de disponíveis nos sites oficiais demoram a chegar até aos profissionais que atuam diretamente com a assistência à saúde.

Trata-se de um assunto de grande relevância não somente para os gestores de saúde de todas as esferas, mas também para todos os profissionais e usuários do sistema público de saúde. Compreendendo, que quanto mais pessoas, principalmente profissionais de saúde, se apropriar desse compromisso assumido pelos CONASS Conselho Nacional dos Secretários Estaduais de Saúde e CONASEMS - Conselho Nacional dos Secretários Municipais de Saúde, quanto mais permeabilidade essa pactuação tiver, maiores serão as chances de se tornar uma política pública efetiva, daí a importância de divulgar tais informações.

O Pacto pela Saúde é um instrumento que vai produzir mudanças significativas nas normativas do Sistema Único de Saúde (SUS) e comporta três dimensões - Pacto pela Vida, Pacto em Defesa do SUS e Pacto de Gestão. Tem como "finalidade a qualificação da gestão pública do SUS, buscando maior efetividade, eficiência e qualidade de suas respostas"(1).

O Sistema Único de Saúde é uma política pública com 20 anos de existência. Nesse período, houve muitos avanços e também desafios. O Pacto pela Saúde 2006, que será anualmente revisado tendo como ênfase as necessidades de saúde da população será o compromisso público, que os gestores do SUS assumirão, de enfrentar desafios e consolidar avanços no fortalecimento do sistema de saúde ${ }^{(2)}$.

O Pacto é resultado de muitas discussões desde 2003, quando o CONASS, solicitou ao Ministério da Saúde - MS revisão dos processos normativos do SUS. Os gestores compreendiam que as normativas do SUS deveriam contemplar a diversidade do Brasil e novas normativas teriam que contemplar os princípios do SUS, sob a égide da responsabilidade sanitária, adequada à realidade de cada Estado e região do país, integrando ações de promoção à saúde, atenção primária, assistência de média e alta complexidade, epidemiologia e controle de doenças, vigilância sanitária e ambiental; a reafirmação da importância das instâncias deliberativas CIB e CIT, e o fortalecimento do controle social ${ }^{(1)}$.

As ações consideradas prioritárias para os secretários foram: compromisso com o SUS e seus princípios; fortalecimento da Atenção Primária; a valorização da saúde; a articulação intersetorial; o fortalecimento do papel dos Estados; a luta pela regulamentação da Emenda Constitucional 29 (que regulamenta os recursos mínimos para o financiamento das ações e serviços públicos de saúde) e por mais recursos financeiros para a área da saúde ${ }^{(3)}$.

Assim sendo, em agosto de 2004, o Ministério da Saúde organizou uma oficina denominada Agenda do Pacto de Gestão que teve como objetivo iniciar a discussão para a "revisão do processo normativo do SUS; e estabelecer as diretrizes, conteúdos e metodologia de trabalho para a elaboração de propostas para pactuação de questões fundamentais para o processo de Gestão do SUS"(1).

Desta oficina participaram representantes do CONASS, CONASEMS e do MS. Depois de mais de dois anos de discussão, em 22 de fevereiro de 2006 o Ministério da Saúde publica a Portaria GM/MS 399 que divulga o Pacto pela Saúde 2006 - Consolidação do SUS e aprova as diretrizes operacionais do referido pacto. Neste contexto, o pacto será revisado a cada ano, visando adaptar-se a realidade enfrentada pelos gestores da saúde, com ênfase nas necessidades de saúde da população(4).

As prioridades estão expressas em objetivos e metas no Termo de Compromisso de Gestão, que é um instrumento público de formalização dos pactos realizados e estão detalhadas no documento denominado Diretrizes Operacionais do Pacto pela Saúde $2006^{(1)}$.

Em abril de 2006, com a publicação da Portaria GM/MS no. 699 acontece a regulamentação das Diretrizes Operacionais dos Pactos pela Vida e de Gestão e a instituição de uma nova forma de transferência de recursos federais destinados ao custeio de ações e serviços de saúde em blocos de financiamento, com a publicação, também, da portaria GM/MS no. $698^{(5-6)}$.

Estas diretrizes vêm salientar a importância da regionalização e de instrumentos de gestão como o Plano Diretor de Regionalização (PDR), Plano Diretor de Investimento (PDI) e a Programação Pactuada e Integrada (PPI). São todos instrumentos que possibilitam a reorganização dos processos de gestão e de regulação do sistema de saúde, buscando a melhoria e qualificação do acesso do usuário do $\operatorname{SUS}^{(1)}$.

\section{AS DI MENSÕES DO PACTO PELA SAÚDE}

O pacto pela saúde tem como pressupostos teóricos alguns conceitos relacionados à construção de rede de assistência por linha de cuidado, baseada na economia de escala e escopo ${ }^{(7)}$ e redes e Pacto Federativo $^{(8)}$. Estas autoras referem esta ser uma 
estratégia para influir nas arenas decisórias de forma organizada em busca da solução de problemas sociais complexos. Compreendem que por meio de redes os atores reconhecem a importância do intercâmbio de recursos técnicos, políticos, informacionais e outros e de negociações em busca de objetivos comuns. Na construção de redes busca-se a difusão do poder e aumento da capacidade estatal na condução de decisões compartilhadas com atores que detêm poderes parciais. No caso do pacto, aponta-se como um espaço de construção de governança os colegiados de gestão regional.

Mendes $^{(7)}$ quando fala em redes refere que as redes de atenção têm três funções: "a função de resolução, a função de coordenação e a função de responsabilização. A função de resolução, intrínseca à sua instrumentalidade como ponto de atenção à saúde, consiste em solucionar a grande e maioria dos problemas de saúde; a função de coordenação, relacionada ao desempenho do papel de centro de comunicação, consiste em organizar os fluxos e contra-fluxos das pessoas e coisas pelos diversos pontos de atenção à saúde da rede; e a função de responsabilização consiste em co-responsabilizar-se pela saúde dos usuários em quaisquer pontos de atenção em que estejam sendo atendidos".

Mendes $^{(8)}$ ainda ressalta que sistemas de informação deficientes; a atenção primária à saúde de baixa qualidade; o protagonismo da atenção especializada; a cultura organizacional com base na autonomia dos serviços; os incentivos econômicos em sentido contrário ao da conformação das redes de atenção à saúde; a debilidade na gestão integrada dos sistemas de saúde; a valorização relativa, pelos usuários, das ações de atenção secundária e terciária; a pouca tradição no uso da gestão da clínica; e a fragilidade dos sistemas logísticos na saúde são barreiras que se apresentam à implantação das redes de atenção à saúde. Todos esses aspectos influenciarão sobremaneira a implantação e implementação dos pactos.

O Pacto pela saúde está organizado em três dimensões. A primeira dimensão é a do Pacto pela vida que estabelece metas sanitárias mobilizadoras, partindo de compromissos sanitários e de gestão que deverão ser atingidos pelo SUS. Está estruturada em uma política de resultados, quando trabalha com a proposição de metas que mostrem a realidade de cada estado ou município, respeitando compromissos orçamentários e financeiros para o alcance desses resultados ${ }^{(1,4-6)}$

O Pacto pela saúde muda radicalmente a forma de pactuação do SUS, pois rompe com os pactos realizados por meio de normas operacionais (Normas Operacionais Básicas - NOB e Norma de Assistência à Saúde - NOAS), que visavam à operacionalização do sistema, distanciando-se, muitas vezes, de compromissos com resultados efetivos. Obriga a repactuação anual e a gestão por resultados sanitários.

Desta forma, as Comissões Intergestores Bipartite - CIB desempenharão um papel preponderante, pois deverão revisar as necessidades e as condições de saúde da população, propondo metas que retratem a realidade de cada Estado.

O Pacto pela Vida destacou como prioridades: a saúde do idoso; o controle do câncer de colo de útero e de mama; o fortalecimento da Atenção Básica/Primária; redução da mortalidade infantil e materna; fortalecimento da capacidade de respostas às doenças emergentes e endemias, com ênfase na dengue, hanseníase, tuberculose, malária e influenza e promoção da saúde ${ }^{(1)}$.

A segunda dimensão é o Pacto em Defesa do SUS. Esta dimensão objetiva discutir a questão da saúde pública e da repolitização do SUS, relembrando os princípios doutrinários da Reforma Sanitária e salientando os direitos garantidos na Constituição (integralidade, eqüidade e universalidade no SUS). Busca consolidar "a política pública de saúde brasileira como uma política de Estado, mais do que uma política de governos"(1). As prioridades são: implementar um projeto permanente de mobilização social com a finalidade, entre outras, de mostrar a saúde como direito de cidadania e o SUS como sistema público universal e elaborar e divulgar a Carta dos direitos dos usuários do $\operatorname{SUS}^{(9)}$, pois a informação é um direito do cidadão e um meio que o indivíduo dispõe para tomar conhecimento e ter poder de determinação a cerca da situação que está vivendo ${ }^{(10)}$.

Neste aspecto, o financiamento da Saúde passa a ser o tema central da discussão. A prioridade do Pacto em Defesa do SUS é a implementação de um projeto de mobilização social com a finalidade de: a) mostrar a saúde como direito de cidadania e o SUS como sistema público universal garantidor desses direitos; b) alcançar, no curto prazo, a regulamentação da Emenda Constitucional no. 29, pelo Congresso Nacional; c) garantir, no longo prazo, o incremento dos recursos orçamentários e financeiros para a saúde; d) aprovar o orçamento do SUS, composto pelos orçamentos das três esferas de gestão, explicitando o compromisso de cada uma delas e e) elaborar e divulgar a carta dos direitos dos usuários do SUS ${ }^{(1)}$.

Quanto à terceira dimensão, o Pacto de Gestão, tem como principal finalidade a busca de maior autonomia aos Estados e aos municípios no que tange aos processos normativos do SUS, definindo responsabilidade sanitária de cada esfera de governo, tornando mais claras as atribuições de cada um, contribuindo assim, para o fortalecimento da gestão compartilhada do SUS. As diretrizes para gestão do 
SUS têm ênfase na Descentralização, Regionalização, Financiamento, Programação Pactuada e Integrada (PPI), Regulação, Planejamento, Gestão do Trabalho e Educação em Saúde, Participação e Controle Social.

Este pacto busca a solidariedade na gestão, avança na regionalização e na descentralização do SUS, quando busca uma diversidade operativa que respeita as peculiaridades de cada região. Reforça, também, a "territorialização da saúde como base para organização dos sistemas, estruturando as regiões sanitárias e instituindo colegiados de gestão regional"(1). Ainda, radicaliza a descentralização de atribuições do Ministério da Saúde para os Estados e para os municípios, promovendo um choque de descentralização, acompanhado da desburocratização dos processos normativos.

\section{O que muda com o Pacto em Saúde}

O pacto não traz grandes mudanças na estrutura de funcionamento da rede de assistência do SUS, porém apresenta componentes novos como o processo de pactuação entre os gestores, extinguindo o processo de habilitação para estados e municípios.

A pactuação preconizada baseia-se principalmente na subsidiariedade, que considerar as possibilidades dos gestores de assumirem responsabilidades, não transferindo para outras esferas a execução de ações que podem ser resolvidas em seu âmbito, com cooperação, coesão regional e responsabilização de cada gestor no seu âmbito de atuação.

Outras mudanças ocorridas foram nas formas de recebimento dos recursos financeiros que passam a ser por blocos de financiamento, além de incentivos financeiros para a área de gestão e planejamento que historicamente nas normas anteriores não foram priorizadas.

A seguir apresentamos algumas diretrizes para a gestão do SUS:

\section{Descentralização}

As diretrizes do pacto de gestão propõem que cada estado defina os modelos organizacionais a serem implantados, desde que pactuados nas CIBs. Assim, pode-se iniciar a descentralização dos processos administrativos relativos à gestão para as Comissões I ntergestores Bipartite.

Neste processo as Secretarias Estaduais em parceria com a representação dos municípios, terão o papel de fortalecer o espaço de negociação e pactuação, por meio de câmaras técnicas e/ou grupos de apoio para dar suporte às decisões da CIB. Criam-se as $\mathrm{ClBs}$ microrregionais. Neste cenário as CIBs estaduais e microrregionais vão ter papel fundamental na "análise da situação de saúde, na elaboração de propostas de intervenção e no estabelecimento dos mecanismos para melhorar a gestão e regulação do sistema"(1).

\section{Regionalização}

A regionalização é pensada como uma estratégia para facilitar e melhorar o acesso aos serviços de saúde, desde que respeitados os conceitos de economia de escala e da qualidade da atenção, buscando o "desenvolvimento de sistemas eficientes e efetivos e criando bases territoriais para o desenvolvimento de redes de atenção à saúde"(1).

Não há a proposição de modelos de região de saúde. A definição desse desenho deverá acontecer em cada CIB e deverá estar de acordo com as necessidades de saúde e a capacidade de oferta das regiões. Este desenho deverá ocorrer sobre territórios sanitários, muito mais do que sobre territórios político-administrativos. Desta forma, "há a necessidade de estudo dos fluxos viários e assistenciais, bem como o uso de metodologias que utilizem os conceitos de economia de escala"(1).

\section{Mecanismos de gestão regional}

O pacto propõe que se criem espaços permanentes de pactuação e co-gestão solidária e cooperativa por meio de um Colegiado de Gestão Regional, ClBs regionais, com a participação de gestores de saúde dos municípios que compõem a Região e da representação estadual.

\section{Financiamento do Sistema Único de Saúde}

A principal mudança no financiamento, relativo ao custeio das ações e serviços de saúde, é a alocação dos recursos federais em cinco blocos, a saber: a) atenção básica/primária; b) atenção de média e alta complexidade; c) vigilância em Saúde; c) assistência farmacêutica e d) gestão do SUS.

A implantação dos pactos será regulamentada por portarias específicas nas CIBs e o instrumento de formalização deste pacto será o Termo de Compromisso de Gestão. Com os Pactos pela Saúde extingue-se o processo de habilitação para estados e municípios preconizados pela NOB SUS 01/96 e pela NOAS SUS 2002. Até que sejam assinados os Termos de Compromisso de Gestão constantes nas diretrizes operacionais do Pacto pela Saúde, mantêm-se as mesmas prerrogativas e responsabilidades dos municípios e estados que estão habilitados em gestão plena $^{(1)}$.

\section{Apresentando uma síntese reflexiva}

Em busca de maior compreensão buscamos identificar como os órgãos colegiados dos Secretários Municipais e Estaduais têm se posicionado referente ao Pacto e como está acontecendo a sua implementação nos Estados Brasileiros.

Estes órgãos assumiram compromisso com o 
Ministério da Saúde em auxiliar a implementação do Pacto em todo o país. O CONASS nos dias 31 de maio e 1 으 de junho, o Seminário Para entender o Pacto pela Saúde 2006, em Brasília. Participaram Secretários e técnicos das Secretarias Estaduais de Saúde (SES). O evento teve como principal objetivo apresentar o pacto e seus pontos mais relevantes para a gestão estadual. O presidente do CONASS, Jurandi Frutuoso (SES/CE), ressaltou a importância da participação do corpo técnico dos Estados no seminário. “É imprescindível nivelar o conhecimento sobre o Pacto, uma vez que o documento rompe com práticas antigas da normatização do SUS"(10).

O tema referente ao Pacto vem sendo pautado pelo CONASEMS, desde o Congresso de Natal em 2004 e o órgão tem estimulado o debate sobre o tema, juntamente com o CONASS e o MS.

Em maio de 2005 durante o XXI Congresso Nacional dos Secretários Municipais de Saúde e II Congresso Brasileiro de Saúde, Cultura de Paz e Não Violência, em Cuiabá, Mato Grosso, foram tomadas deliberações referentes ao Pacto, sendo incorporadas na agenda das três esferas de governo, aprovados os seus princípios e cronograma de implantação.

Em junho de 2006, em Recife, estado de Pernambuco, os Secretários Municipais de Saúde reunidos, no XXII Congresso Nacional das Secretarias Municipais de Saúde, III Congresso Brasileiro de Saúde, Cultura de Paz e Não Violência e V Congresso da Rede Américas, reiteram seu compromisso com a melhoria da qualidade de vida e da saúde de todos os brasileiros e demais povos do continente americano, bem como com a consolidação do Sistema Único de Saúde (SUS), sendo aprovada a "Carta de Recife" que apresenta as deliberações que deverão nortear a atuação do CONASEMS, dos Conselho dos secretários Municipais de Saúde - COSEMS e das Secretarias Municipais de Saúde(11).

Novamente, se afirma a condição para a implantação desta nova proposta de reestruturação do SUS. Entretanto, para que a informação sobre o pacto chegue a cada Secretaria Estadual e municipal em todo o território, o Ministério conta com a parceria dos órgãos colegiados dos secretários para esta disseminação. No entanto, se observa que a mesma está sendo implantada de forma singular em cada estado brasileiro. O Ministério tem colocado o Departamento de Apoio Integrado a Descentralização (DAD) para auxiliar Estados e Municípios na sua implementação, mas este processo vem ocorrendo diferenciadamente em cada Estado, sendo que na reunião de dezembro foi aprovada na CIT as primeiras adesões ao Pacto (Estado do Maranhão e Ceará), e dos municípios da (Bahia, Ceará, Maranhão, Pernambuco e São Paulo). Como a sua implementação ainda está lenta um fator que influenciou a implantação do pacto é o fato de ter coincidido com período eleitoral, o que dificultou a pactuação com os gestores. A dificuldade de implantação depende em parte do entendimento do processo de gestão dos recursos financeiros e das relações de poder sempre presentes em qualquer iniciativa no âmbito da gestão pública.

Quanto à adesão do município de São Luiz, o secretário de Saúde de São Luiz e também presidente do CONASEMS, Edmundo Costa Gomes, comemorou o fato de seu município, ser a primeira capital a assinar o Termo de Compromisso de Gestão do Pacto. Para a capital a adesão ao Pacto é importante, pois mostra o compromisso da gestão da saúde do município ao SUS. Em 2008, transcorrido o processo inicial de adesão, temos o seguinte quadro, segundo informações do Departamento de Apoio á Descentralização do Ministério da Saúde, 20 estados já têm seus termos homologados pela $\mathrm{CIT}, 4$ (quatro) aguardam homologação, restando apenas 6 (seis) estados aderirem. Com relação aos municípios, dos 5563 municípios brasileiros, 2.466 (43\%) já aderiram. No Estado de Santa Catarina foram realizados seminários em conjunto SES e COSEMS para e assimilação e divulgação do conteúdo do Pacto, visando definir uma agenda de trabalho conjunta. A adesão no estado ainda está lenta, possuindo apenas 23 municípios com homologação, porém o número de municípios que já está encaminhando documento para o seu ingresso, aponta para um avanço destes números ${ }^{(12)}$.

Sabemos que este processo ainda está sendo construído, porém cabe destacar alguns consensos que requerem a vigilância e participação de gestores, trabalhadores e usuários do SUS que são: o estabelecimento de metas sanitárias; mudanças no modelo de atenção à saúde; organização em rede de atenção à saúde; articulação em diferentes espaços territoriais; garantia do acesso aos serviços de boa qualidade; coerência com os princípios do SUS; responsabilidade sanitária e solidária; adequação à realidade de cada região e integração das ações e dos serviços ${ }^{(13)}$.

Contudo, apresenta-se como principal desafio implementar os pressupostos desse pacto, quais sejam: a) subsidiariedade, que deve considerar as possibilidades dos gestores de assumirem responsabilidades, não transferindo para outras esferas a execução de ações que podem ser resolvidas em seu âmbito; b) cooperação, ou seja, “a criação de mecanismos institucionais que promovam a articulação e a pactuação para o desenvolvimento das ações e serviços de natureza regional, inclusive co-financiamento"(13); c) coesão regional como o reconhecimento que a ação regional deriva de um processo político com a participação de todos os envolvidos no processo de regionalização; d) responsabilização, gestores estabelecendo 
compromissos entre si na busca da resolutividade dos problemas de saúde da população em um dado território e e) territorialização, a pactuação de territórios levando em consideração as peculiaridades locais e as regiões de saúde existentes ${ }^{(13)}$.

\section{APONTANDO ALGUNS QUESTIONAMENTOS À GUISA DE CONCLUSÃO}

Muitos ainda são os questionamentos a respeito desses pactos e, já no processo de discussão, em seminários com gestores e oficinas promovidas pelo Ministério da Saúde, os aspectos levantados chamavam a atenção pela repetição de prioridades pactuadas no Pacto pela Vida, pois além de continuar a atender a mulher em ações parcelares, tais como: controle do câncer em colo de útero e mama, redução da mortalidade materna, esquecendo a tão propalada integralidade. Apesar de argumentarem que esses eixos foram repetidos por terem baixa cobertura, fácil resolubilidade e estratégias bem conhecidas e de baixo custo. Em relação à saúde do idoso, adverte-se sobre a falta de detalhamento do projeto, incluindo custos, sobre que atenção diferenciada e mudança no acolhimento está se falando, bem como que melhoria na assistência farmacêutica se quer.

No Pacto de Gestão aparece como principal componente o fortalecimento das $\mathrm{CIBs} e$ dos colegiados regionais. Será que COSEMS e Secretarias de Estado querem dividir este poder? Os Colegiados Regionais não fortaleceriam a regionalização e a gestão do sistema?

Os gestores devem pensar de forma solidária quando fizerem seus planejamentos, olhando ao seu redor, buscando atender aos seus vizinhos que não tiverem ainda alcançado seu estágio nos Termos de Compromisso de Gestão.

Considerando que a principal mudança no financiamento é a criação de blocos de financiamento, então a criação de cinco blocos para financiamento flexibiliza a gestão financeira? Como concretizar este processo se não vem recurso financeiro novo? Como as vigilâncias sanitária e epidemiológica vão gerir seus recursos agora?

Com relação à adesão ao pacto, qual é o tempo que os municípios terão para assinar o Termo de Compromisso de Gestão? Que prazo terão para executar as ações e irem agregando outras? Não serão muitos pactos e pouco impacto para a situação de saúde no Brasil? Como os municípios irão contratualizar seus prestadores de serviços? Todos os municípios não podem ser plenos em tudo? Como ficaria então? Como estabelecer a regionalização? Considera-se que a contratualização é o maior avanço neste processo, apesar de ser o que possui maior resistência. Desde a implantação do SUS esta é a estratégia que menos avançou, pois ainda se mantem contratos antigos e com valor legal discutível, sendo alguns ainda do antigo INAMPS. No pacto, os municípios e o Estado, terão que refazer todos os seus contratos com os prestadores do SUS ambulatoriais e hospitalares, dentro do limite que cada um tem.

Será que acontecerá mesmo uma priorização e estruturação na área de recursos humanos já que a área figura entre as prioridades, principalmente nas capitais e cidades com mais de 500 postos de trabalho públicos?

Muitas são ainda as dúvidas existentes, entretanto há a necessidade de implementar o processo visando à continuidade e o fortalecimento do Sistema Único de Saúde, implementando as diretrizes, iniciativas e ações do Pacto em Defesa do SUS.

Apesar de parecer complexo o processo, é imperioso que seja rapidamente socializado e compreendido para que estes instrumentos venham fortalecer a gestão do SUS. Entretanto, deve-se compreender que os pactos dependem de relacionamento entre os vários níveis de governo e os interesses da Federação, fomentando a convivência e a interdependência. Neste sentido, os pactos devem ser realizados de forma clara entre as partes e compreende-se que a decisão de aderir envolve muitos aspectos tais como: decisão política, estrutura técnico-administrativa e fiscal, além de recursos humanos capacitados e compromissados com a gestão pública para assumir essa pactuação. No entanto, para o avanço desse processo torna-se necessário que estados e municípios adiram ao pacto. Compreende-se que a decisão de aderir envolvem muitos aspectos, tais como: disponibilidade de recursos administrativos e fiscais e a disposição de enfrentamento a difíceis negociações entre poderes muito desiguais e uma coordenação do governo federal, tradicionalmente frágil. No entanto, é um processo que precisa ser aprendido e compartilhado por todos os envolvidos, pois esta é a forma que se apresenta a política pública de saúde em busca de resultados sanitários efetivos para as necessidades de saúde da população.

\section{REFERÊNCIAS}

1. Conselho Nacional de Secretários de Saúde (CONASS). Nota Técnica n. 13/06. Para entender o Pacto pela Saúde 2006 - Financiamento. Portaria 698/GM de 30/03/2006. Volume IV [Internet]. Brasília (Brasil): CONASS; 2006. Available from: http://www.conass.org. br/admin/arquivos/NT\% 201306.pdf

2. Deus J. Pacto visa o fortalecimento do SUS em todo estado de Mato Grosso. Diário de Cuiabá [Internet]. 2006 Apr 25 [cited 2009 feb 16]. Saúde. Available from: 
http://www.diariodecuiaba.com.br/detalhe.php?cod= 251738.

3. Carvalho G. Pactos do SUS - 2005 - Comentários Preliminares [Internet]. Campinas: Instituto de Direito Sanitário Aplicado; 2005 Nov 15 [cited 2009 mar 11]. Available from: http://www.idisa.org. br/site/artigos/visualiza_conteu dol. $p h p ? i d=1638$

4. Ministério da Saúde. Referido Pacto. Brasília: Ministério da Saúde; 2006.

5. Ministério da Saúde. Portaria GM 698 de 30 de março de 2006. Define o Financiamento tripartite no custeio das ações de saúde. Brasília (Brasil): Ministério da Saúde; 2006.

6. Ministério da Saúde. Portaria GM 699 de 22 de março de 2006. Aprova e regulamenta o pacto pela saúde. Brasília (Brasil): Ministério da Saúde; 2006.

7. Mendes EV. A modelagem das redes de atenção à saúde. Belo Horizonte: Secretaria de Estado da Saúde de Minas Gerais; 2002.

8. Mendes EV. Revisão bibliográfica sobre redes de atenção à saúde. Belo Horizonte: Secretaria de Estado da Saúde de Minas Gerais; 2007.

9. Ministério da Saúde. Carta dos direitos dos usuários da saúde. Brasília: Ministério da Saúde; 2006.

10. Lunardi VL, Costa VT, Chaves PL. A enfermagem frente aos direitos de pacientes hospitalizados. Texto contexto-enferm. 2005; 14(1): 38-43.

11. Carta de Recife. RADIS - comunicação em saúde [Internet]. 2006 [cited 2009 mar 12]; (48). Avaiable from: http://www4.ensp.fiocruz.br/radis/48/web02.html

12. CooperaSUS [Internet]. Brasília: Ministério da Saúde (BR) [cited 2009 feb 16]. Última reunião tripartite de 2006 fecha o ano com importantes pactuações. Available from: https://200.214.130.60/cooperasus/boletim/bv15/m at01.htm.

13. Conselho Nacional de Secretarias Municipais de Saúde [Internet]. Brasília: Ministério da Saúde (BR) [update 2007 jul 02; cited 2009 mar 11]. Pacto pela Vida, em Defesa do SUS e de Gestão. Available from: http://www. conasems. org. br/files/dia27/Of2Construi ndoMinisterioSaude.pdf.

Artigo recebido em 05.12.07.

Aprovado para publicação em 31.03.09. 